\title{
Antiretroviral treatment and expression of the mRNA levels for Pgp, MRP1, MRP4 and MRP5 in HIV antiretroviral naïve patients. Follow-up at 48 weeks
}

\author{
Francesca Falasca', Francesca Graziano', Laura Antonelli, Paola Maida', Claudia Montagna', Bambina Rizzo', \\ Vincenzo Renda ${ }^{2}$, Guido Antonelli ${ }^{1}$, Ombretta Turriziani ${ }^{1 *}$
}

From $16^{\text {th }}$ International Symposium on HIV and Emerging Infectious Diseases Marseille, France. 24-26 March 2010

\section{Background}

The ATP-binding cassette genes represent the largest family of transmembrane proteins [including multidrug resistant proteins (MRPs) and P-glycoprotein (Pgp)] able to drive the transport of various molecules across cell membranes. Several studies have demonstrated that most of the above transporter are also able to transport antiretrovirals.

The aim of this study was to evaluate whether the antiretroviral treatment might affect the mRNA expression of Pgp and some MRPs.

\section{Methods}

Blood samples were collected from 13 HIV-positive patients treatment naïve. After the beginning of the treatment, samples were collected at 12, 24, 36 and 48 weeks. Eight patients were treated with Kaletra and Truvada (group I) and five patients with Efavirenz and NRTIs (truvada or combivir) (Gropu II). Expression of mRNA of the Pgp, MRP1, MRP4, and MRP5 was evaluated by real-time-PCR using the TaqMan technology (ABI Prism 7000; Applied Biosystems).

\section{Results}

MDR1 and MRP4 expression was not affected by treatment with PI and NRTI. In fact, at all time analyzed the mRNA levels of these transporters did not significantly differed from the mRNA levels detected before the beginning of treatment. As far as MRP1 and MRP5 are concerned, a modest, but not significant, reduction in the mRNA expression levels was observed after beginning of treatment. In patients belonging to the group II basically the same results were obtained. Looking at the individual trend of the mRNA expression of the above transporters in each patient it can be seen that the expression levels of these transporters seems to change during follow up but it is independent of type and time of treatment. The expression of the mRNA levels of these transporters appears to increase in some patients and decrease in other individuals suggesting that a high interindividual variability in the modulation of these mRNA does exist.

\section{Discussion}

Antiretroviral treatment does not significantly affect the expression levels of mRNA of transported analyzed. However an interindividual variability in the expression of these mRNA has been documented during the follow up and further studies are needed to evaluate whether the over-expression of these mRNA may affect the success of therapy.

\section{Author details}

'Department of Experimental Medicine, Virology Section, Sapienza University, Rome, Italy. ${ }^{2}$ Department of Infectious Diseases Umberto I Hospital, Rome, Italy

Published: 11 May 2010

\section{doi:10.1186/1742-4690-7-S1-P42}

Cite this article as: Falasca et al:: Antiretroviral treatment and expression of the mRNA levels for Pgp, MRP1, MRP4 and MRP5 in HIV antiretroviral naïve patients. Follow-up at 48 weeks. Retrovirology 2010 7(Suppl 1):P42.

* Correspondence: ombretta.turriziani@uniroma1.it

${ }^{1}$ Department of Experimental Medicine, Virology Section, Sapienza University, Rome, Italy 